\title{
Some Thoughts on the Impact of COVID-19 on Plastic Surgery
}

\author{
Pengfei Sun ${ }^{1} \cdot$ Yanjin Wang ${ }^{2} \cdot$ Huachang Sun $^{3} \cdot$ Fang Luan $^{3}$
}

Received: 13 September 2020/Accepted: 13 September 2020/Published online: 25 September 2020

(C) Springer Science+Business Media, LLC, part of Springer Nature and International Society of Aesthetic Plastic Surgery 2020

Level of Evidence $V$ This journal requires that authors assign a level of evidence to each article. For a full description of these Evidence-Based Medicine ratings, please refer to the Table of Contents or the online Instructions to Authors www.springer.com/00266.

\section{Dear Editor}

The outbreak of coronavirus disease (COVID-19) in December 2019 changed the way human society operates [1]. As plastic surgeons, we are worried about the future of plastic surgery in the context of the COVID-19 epidemic. We read the published study by Kaye and Paprottka entitled Elective, Non-urgent Procedures and Aesthetic Surgery in the Wake of SARS-COVID-19: Considerations Regarding Safety, Feasibility and Impact on Clinical Management [2],carefully. Due to our in-depth study of COVID-19 in the early stages of the epidemic [3-5], we strongly agree with the following points mentioned in the article.

Fang Luan

zbzblff@163.com

1 Department of Auricular Reconstruction, Plastic Surgery Hospital, Peking Union Medical College and Chinese Academy of Medical Sciences, No. 33 Bada Road, Shijingshan District, Beijing 100144, People's Republic of China

2 Department of Plastic Surgery, Affiliated Hospital of Qingdao University, No. 16 Jiangsu Road, Qingdao, Shandong Province, People's Republic of China

3 Department of Plastic Surgery, Zibo Central Hospital, No. 54 The Communist Youth League Road, Zibo, Shandong Province, People's Republic of China
1. In the near future, several pandemics of COVID-19 are inevitable around the world [6]. We believe that the end result of COVID-19 will be a decline in virulence, a decline in mortality among patients infected with COVID-19, and COVID-19 will coexist with humans, just as the influenza virus does [7]. After several outbreaks of COVID-19, some humans will produce antibodies to the COVID-19.

2. At present, the conclusions of studies that COVID-19 increases mortality in surgical patients are not fully applicable to plastic surgery because plastic surgery is mostly less traumatic surgery for patients and the group of patients receiving plastic surgery is younger, with less suffering from chronic diseases such as diabetes and hypertension. Therefore, we believe that plastic surgery with a short duration and less physical trauma can be performed in the context of the COVID19 epidemic.

3. With regard to the perioperative standard use of antibiotics mentioned by the authors in the article, we believe that prophylactic use of antibiotics can be avoided in plastic surgery without obvious infection lesions if the operation time is less than $3 \mathrm{~h}$. Plastic surgery with a clean incision within $3 \mathrm{~h}$ has a low chance of bacterial infection, and there is no evidence that prophylactic use of antibiotics can help prevent infection with COVID-19.

4. It is essential that every patient is treated as a COVID19-infected patient. In the process of receiving patients, plastic surgery, communication with patients and adequate protection can avoid the infection of COVID-19 to the maximum extent. Every patient who comes to plastic surgery should be screened for 
COVID-19 and should be highly alert to asymptomatic infection with COVID-19.

Although there are many points that we agree with in the author's article, there are also many points that make us confused.

1. The authors mentioned vitamin $C$, vitamin $D$, zinc, curcumin and other drugs in the article. They believe that these drugs can enhance the body's resistance to prevent or treat the COVID-19. We question the author on this point of view. COVID-19 infects humans by binding to ACE2 receptors, and the main cause of severe illness and even death in humans is the factor storm caused by the COVID-19 infection in humans [8]. We don't think the more resistant the body is, the more resistant it is to avoid infection with COVID-19. There is also no clear evidence that these drugs can effectively reduce the infection of the COVID-19 and improve the survival rate of patients infected with the COVID-19.

2. In this paper, the author describes the operation of the anesthesiologist to prevent COVID-19 infection during anesthesia, but does not describe in detail how plastic surgeons prevent COVID-19 infection during surgery. We believe that it is necessary for plastic surgeons to wear surgical masks and eye masks during the operation. Non-facial surgery can also place a plastic closure mask on a patient's face to prevent respiratory droplet and aerosol transmission of COVID-19. Blood spatter should also be avoided during surgery.

3. Vaccinating every plastic surgeon may be a reliable way to prevent COVID-19 infection. But the COVID19 mutates quickly [9], and some COVID-19 patients who have been cured become infected again [10]. Therefore, we are concerned about the method of vaccination against COVID-19 infection.

In the context of the COVID-19 virus epidemic, how to carry out aesthetic plastic surgery safely and efficiently and how to ensure the safety of patients and medical personnel, these are the great challenges that each of our plastic surgeons are facing now. We believe that with the deepening of the understanding of the new coronary pneumonia virus, we will be able to find an effective way to prevent and treat the COVID-19.

\section{Compliance with Ethical Standards}

Conflict of interest The authors declare that they have no conflict of interest.

Human and Animal Participants This article does not contain any studies with human participants or animals performed by any of the authors.

Informed Consent For this type of study, informed consent is not required.

\section{References}

1. Buder F, Hitzenbichler F, Ehrenstein B et al (2020) The outbreak of COVID-19 in China. Internist (Berl) 61(8):776-781

2. Kaye K, Paprottka F, Escudero R et al (2020) Elective, nonurgent procedures and aesthetic surgery in the wake of SARSCOVID-19: considerations regarding safety, feasibility and impact on clinical management. Aesth Plast Surg 44(3):1014-1042

3. Sun P, Lu X, Xu C, Sun W et al (2020) Understanding of COVID-19 based on current evidence. J Med Virol 92(6):548-551

4. Sun P, Qie S, Liu Z et al (2020) Clinical characteristics of hospitalized patients with SARS-CoV-2 infection: a single arm metaanalysis. J Med Virol 92(6):612-617

5. Sun P, Lu X, Xu C et al (2020) CD-sACE2 inclusion compounds: an effective treatment for coronavirus disease 2019 (COVID-19). J Med Virol. https://doi.org/10.1002/jmv.25804

6. Konda M, Dodda B, Konala VM et al (2020) Potential Zoonotic Origins of SARS-CoV-2 and insights for preventing future pandemics through one health approach. Cureus 12(6):e8932

7. Chen P, Zeng Z, Du H (2020) Establishment and validation of a drug-target microarray for SARS-CoV-2. Biochem Biophys Res Commun 530(1):4-9

8. Lutz C, Maher L, Lee C et al (2020) COVID-19 preclinical models: human angiotensin-converting enzyme 2 transgenic mice. Hum Genom 14(1):20

9. Meza-Robles C, Barajas-Saucedo CE, Tiburcio-Jimenez D et al (2020) One-step nested RT-PCR for COVID-19 detection: a flexible locally developed test for SARS-CoV2 nucleic acid detection. J Infect Dev Ctries 14(7):679-684

10. To KK, Hung IF, Ip JD et al (2020) COVID-19 re-infection by a phylogenetically distinct SARS-coronavirus-2 strain confirmed by whole genome sequencing. Clin Infect Dis. https://doi.org/10. 1093/cid/ciaa1275

Publisher's Note Springer Nature remains neutral with regard to jurisdictional claims in published maps and institutional affiliations. 